Research Article

\title{
Upper domatic number of regular graphs
}

\author{
Libin Chacko Samuel*, Mayamma Joseph \\ Department of Mathematics, CHRIST (Deemed to be University), Bangalore, Karnataka, India
}

(Received: 29 May 2021. Received in revised form: 21 August 2021. Accepted: 22 September 2021. Published online: 29 September 2021.)

(c) 2021 the authors. This is an open access article under the CC BY (International 4.0) license (www.creativecommons.org/licenses/by/4.0/).

\begin{abstract}
A partition $\pi=\left\{V_{1}, V_{2}, \ldots, V_{k}\right\}$ of the vertex set $V(G)$ of a graph $G=(V, E)$ is an upper domatic partition if $V_{i}$ dominates $V_{j}$ or $V_{j}$ dominates $V_{i}$ or both for all $V_{i}, V_{j} \in \pi$. The maximum order of an upper domatic partition of $G$ is called the upper domatic number $D(G)$ of $G$. In this article, we determine the upper domatic number of 4-regular graphs. We also find the upper domatic number of 5-regular graphs with girth at least five and determine the upper domatic number of the complements of cycles.
\end{abstract}

Keywords: domatic number; $r$-regular graphs; upper domatic number.

2020 Mathematics Subject Classification: 05C69.

\section{Introduction}

Graph colouring and domination are two well-studied concepts in graph theory. Connecting these two concepts, Cockayne and Hedetniemi initiated the study of the domatic number in 1977 [1]. The domatic number, denoted by $d(G)$, is the maximum number of sets in a partition of the vertex set into mutually disjoint dominating sets. For two disjoint sets $A$ and $B$ of vertices, set $A$ dominates set $B$, denoted by $A \rightarrow B$, if each vertex in $B$ is adjacent to at least one vertex in $A$, else we write $A \nrightarrow B$. A subset $S$ of $V(G)$ is a dominating set of $G$ if $S$ dominates $V(G) \backslash S$. Exploring the relation between sets in a vertex partition, Haynes et al. [3] introduced the concept of upper domatic number $D(G)$, which is the maximum cardinality of a vertex partition $\pi=\left\{V_{1}, V_{2}, \ldots, V_{k}\right\}$ of a graph $G$ such that for each pair of sets $V_{i}, V_{j} \in \pi, V_{i} \rightarrow V_{j}$ or $V_{j} \rightarrow V_{i}$ or both. An upper domatic partition of $G$ with cardinality $D(G)$ is referred to as a $D$-partition of $G$.

Throughout this paper, we consider only finite, simple and undirected graphs $G=(V, E)$ of order $n=|V|$ and size $m=|E|$. For the notations and terminologies not defined here, we refer the reader to $[2,4,6]$. The degree of a vertex $v \in V$, denoted by $\operatorname{deg}(v)$, is the number of vertices adjacent to $v$. The maximum (minimum) degree of a vertex in a graph $G$ is denoted by $\Delta(G)(\delta(G))$. The set of vertices at distance equal to $k$ from the vertex $u$ is represented by $N^{k}(u)$. Paths, cycles and complete graphs of order $n$ are denoted by $P_{n}, C_{n}$ and $K_{n}$, respectively. A maximal complete subgraph of a graph is called clique. The maximum order of a clique of $G$ is called the clique number $\omega(G)$.

Let $\pi=\left\{V_{1}, V_{2}, \ldots, V_{k}\right\}$ be a vertex partition of a graph $G$. A set $V_{i} \in \pi$ is a source set if $V_{i}$ is a dominating set of $G$ and a sink set if $V_{j} \rightarrow V_{i}$, for all $1 \leq j \leq k$. For a subset $S$ of $V(G), G[S]$ represents the subgraph of $G$ induced by $S$. An $r$-regular graph is a graph with every vertex having degree $r$.

In this article, we study the upper domatic number of $r$-regular graphs and settle the problem in the special case when $r=4$. The upper domatic number of 5-regular graphs with girth at least five also is determined. Further we find the upper domatic number of complements of cycles.

\section{Main results}

For small values of $r$, the upper domatic number of an $r$-regular graph is determined in [3].

Theorem 2.1. [3] For any $r$-regular graph $G$ where $r \in\{0,1,2,3\}, D(G)=r+1$.

Haynes et al. also gave an upper bound for the upper domatic number of any graph in terms of its maximum degree.

Theorem 2.2. [3] For a graph $G$ with maximum degree $\Delta(G), D(G) \leq \Delta(G)+1$.

\footnotetext{
*Corresponding author (libin.samuel@res.christuniversity.in).
} 
Another upper bound for the upper domatic number in terms of the order and clique number of the graph is presented in [5].

Theorem 2.3. [5] For any graph $G$ with clique number $\omega(G), D(G) \leq \frac{n+\omega(G)}{2}$.

We next present the following sufficiency condition for a 4-regular graph to have an upper domatic number of five.

Theorem 2.4. For any 4-regular graph $G, D(G)=5$ if $G$ contains a cubic graph as an induced subgraph.

Proof. Consider a 4-regular graph $G$ having a 3-regular induced subgraph, say $H$. By Theorem 2.1, $D(H)=4$. Moreover, $H$ being a 3-regular graph, every vertex in $V(H)$ is adjacent to exactly one vertex in $V(G) \backslash V(H)$, implying that $V(G) \backslash V(H) \rightarrow$ $V(H)$. Hence, an upper domatic partition of order five can be obtained by adding the set $V(G) \backslash V(H)$ to a $D$-partition of $H$. Therefore, by Theorem 2.2, $D(G)=5$.

Corollary 2.1. If a graph $G$ contains a cubic induced subgraph and $\delta(G) \geq 4$, then $D(G) \geq 5$.

Corollary 2.2. For any 4-regular graph $G, D(G)=5$ if $G$ contains $K_{4}$.

The result in Corollary 2.2 can be generalised for any $r$-regular graph containing $K_{r}$.

Theorem 2.5. For any r-regular graph $G, D(G)=r+1$ if $G$ contains $K_{r}$.

It is evident from the literature that for a 4-regular graph $G, 4 \leq D(G) \leq 5$. Next, we characterise 4-regular graphs having $D(G)=4$. Recall that the complete multipartite graph with $k$ vertices each in $t$ partite sets is denoted by $G_{t, k}$ in [3], for example the complete tripartite graph $G_{3,2}$ with three independent partite sets $\left\{u_{1}, u_{4}\right\},\left\{u_{2}, u_{5}\right\}$ and $\left\{u_{3}, u_{6}\right\}$ in Figure 1 .

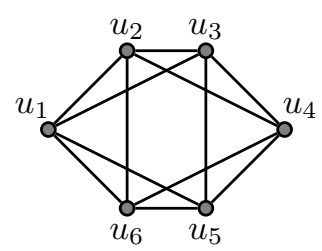

Figure 1: The graph $G_{3,2}$.

Theorem 2.6. For a 4-regular connected graph $G, D(G)=4$ if and only if $G=G_{3,2}$.

Proof. Consider the 4-regular graph $G_{3,2}$ with vertex labelling as shown in Figure 1. The partition $\pi=\left\{\left\{u_{4}, u_{5}, u_{6}\right\},\left\{u_{1}\right\}\right.$, $\left.\left\{u_{2}\right\},\left\{u_{3}\right\}\right\}$ is an upper domatic partition, implying that $D\left(G_{3,2}\right) \geq 4$. If $D\left(G_{3,2}\right)>4$, then any $D$-partition of $G_{3,2}$ contains at least four singleton sets, which is impossible as $\omega\left(G_{3,2}\right)=3$. Hence, $D\left(G_{3,2}\right)=4$.

Now assume that $G$ is a connected 4-regular graph with $D(G)=4$. Assume that the graph $G$ is different from $G_{3,2}$. We will prove that $D(G) \geq 5$. Consider a vertex, say $u_{1} \in V(G)$. Since $G$ is 4-regular, there are 11 possible induced subgraphs $G\left[N\left[u_{1}\right]\right]$, as shown in Figure 2.

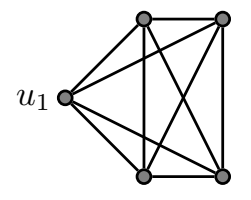

$\mathcal{G}_{1}$

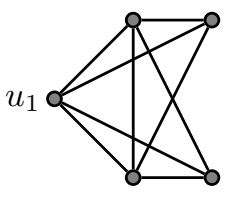

$\mathcal{G}_{2}$

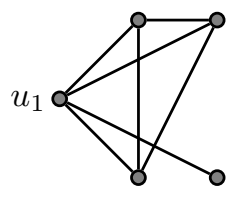

$\mathcal{G}_{3}$

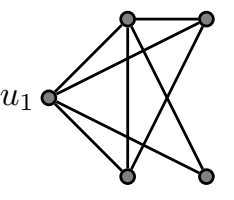

$\mathcal{G}_{4}$

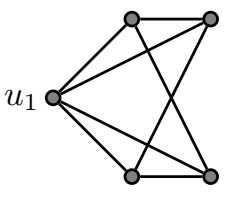

$\mathcal{G}_{5}$

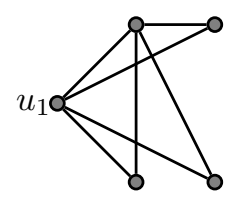

$\mathcal{G}_{6}$

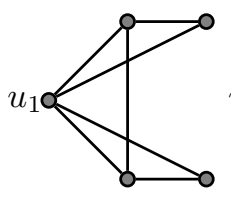

$\mathcal{G}_{7}$

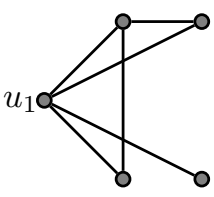

$\mathcal{G}_{8}$

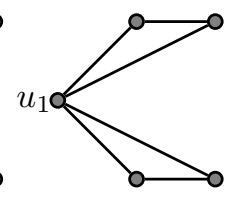

$\mathcal{G}_{9}$

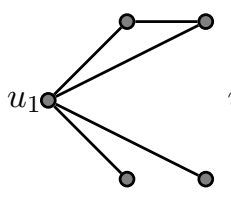

$\mathcal{G}_{10}$

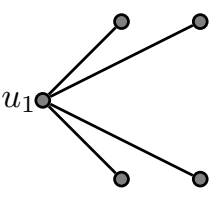

$\mathcal{G}_{11}$

Figure 2: Graphs of order five with a dominating vertex.

Case 1: $G\left[N\left[u_{1}\right]\right] \in\left\{\mathcal{G}_{1}, \mathcal{G}_{2}, \mathcal{G}_{3}, \mathcal{G}_{4}\right\}$.

Since each of $\mathcal{G}_{1}, \mathcal{G}_{2}, \mathcal{G}_{3}$ and $\mathcal{G}_{4}$ contains an induced $K_{4}$, from Corollary 2.2 it follows that $D(G)=5$. 
Case 2: $G\left[N\left[u_{1}\right]\right]=\mathcal{G}_{5}$, as shown in Figure 3 .

In this case we observe that $\left|N^{2}\left(u_{1}\right)\right|>1$, else $G$ is isomorphic to $G_{3,2}$. Therefore, there exist at least two vertices, say $u_{6}, u_{7} \in N^{2}\left(u_{1}\right)$. Suppose we have a pair of adjacent vertices, say $u_{2}, u_{3} \in N^{1}\left(u_{1}\right)$ such that $u_{2}$ is adjacent to $u_{6}$ and $u_{3}$ is adjacent to $u_{7}$. Then, $\pi=\left\{\left\{u_{1}\right\},\left\{u_{2}\right\},\left\{u_{3}\right\},\left\{u_{4}, u_{7}\right\},\left\{u_{5}, u_{6}\right\} \cup V^{\prime}\right\}$, where $V^{\prime}=V(G) \backslash\left\{u_{1}, u_{2}, \ldots, u_{7}\right\}$, is an upper domatic partition of $G$. In the case of a non-adjacent pair of vertices, say $u_{2}, u_{5} \in N^{1}\left(u_{1}\right)$ having adjacency between the pair of vertices $\left(u_{2}, u_{6}\right)$ and $\left(u_{5}, u_{7}\right)$, the vertex $u_{3}$ is adjacent either to $u_{6}$ or to another vertex $u_{8} \in N^{2}\left(u_{1}\right)$. In the former case $\left\{\left\{u_{1}\right\},\left\{u_{3}\right\},\left\{u_{5}\right\},\left\{u_{4}, u_{6}\right\},\left\{u_{2}, u_{7}\right\} \cup V^{\prime}\right\}$, where $V^{\prime}=V(G) \backslash\left\{u_{1}, u_{2}, \ldots, u_{7}\right\}$ forms an upper domatic partition of $G$, whereas in the latter case $\left\{\left\{u_{1}\right\},\left\{u_{3}\right\},\left\{u_{5}\right\},\left\{u_{4}, u_{8}\right\},\left\{u_{2}, u_{7}, u_{6}\right\} \cup V^{\prime}\right\}$, where $V^{\prime}=V(G) \backslash\left\{u_{1}, u_{2}, \ldots, u_{8}\right\}$ constitute an upper domatic partition of $G$. Hence, $D(G)=5$.

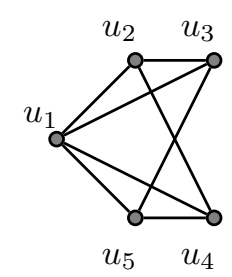

Figure 3: The graph $\mathcal{G}_{5}$.

Case 3: $G\left[N\left[u_{1}\right]\right]=\mathcal{G}_{6}$, as shown in Figure 4 .

From the structure of $\mathcal{G}_{6}$, we note that there exist vertices $u_{6}, u_{7} \in N^{2}\left(u_{1}\right)$ that are adjacent to $u_{3}$. Then, the sets $\left\{u_{1}\right\},\left\{u_{2}\right\},\left\{u_{3}\right\},\left\{u_{4}, u_{7}\right\}$ along with $V^{\prime}=V(G) \backslash\left\{u_{1}, u_{2}, u_{3}, u_{4}, u_{7}\right\}$ constitutes an upper domatic partition of cardinality five, proving that $D(G)=5$.

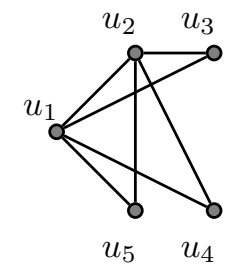

Figure 4: The graph $\mathcal{G}_{6}$.

Case 4: $G\left[N\left[u_{1}\right]\right] \in\left\{\mathcal{G}_{7}, \mathcal{G}_{8}\right\}$, as shown in Figure 5.

When $G\left[N\left[u_{1}\right]\right]=\mathcal{G}_{7}$ or $\mathcal{G}_{8}$, there is a vertex $u_{6} \in N^{2}\left(u_{1}\right)$ such that $u_{3}$ is adjacent to $u_{6}$ but $u_{2}$ is not adjacent to $u_{6}$. Then $\pi=\left\{\left\{u_{1}\right\},\left\{u_{2}\right\},\left\{u_{3}\right\},\left\{u_{5}, u_{6}\right\},\left\{u_{4}\right\} \cup V^{\prime}\right\}$, where $V^{\prime}=V(G) \backslash\left\{u_{1}, u_{2}, \ldots, u_{6}\right\}$, is an upper domatic partition of $G$ proving that $D(G)=5$.
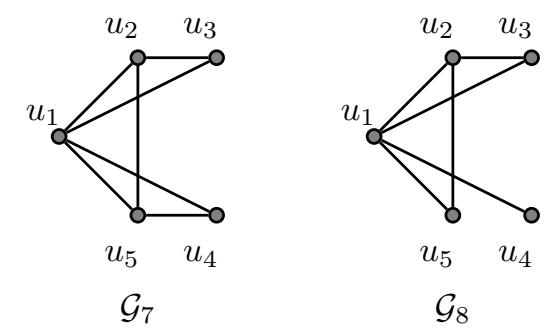

Figure 5: The graphs $\mathcal{G}_{7}$ and $\mathcal{G}_{8}$.

Case 5: $G\left[N\left[u_{1}\right]\right] \in\left\{\mathcal{G}_{9}, \mathcal{G}_{10}\right\}$, as shown in Figure 6 .

In this case, suppose there is a vertex $u_{6} \in N^{2}\left(u_{1}\right)$ such that $u_{2}$ and $u_{3}$ are adjacent to $u_{6}$. Then considering $V^{\prime}=V(G) \backslash\left\{u_{1}, u_{2}, \ldots, u_{6}\right\}$, we get an upper domatic partition $\pi=\left\{\left\{u_{1}\right\},\left\{u_{2}\right\},\left\{u_{3}\right\},\left\{u_{4}, u_{6}\right\},\left\{u_{5}\right\} \cup V^{\prime}\right\}$ of $G$ proving that $D(G)=5$. Else, there exist vertices $u_{6}, u_{7} \in N^{2}\left(u_{1}\right)$ such that $u_{2}$ is adjacent to $u_{6}$ and $u_{3}$ is adjacent to $u_{7}$. Then $\pi=\left\{\left\{u_{1}\right\},\left\{u_{2}\right\},\left\{u_{3}\right\},\left\{u_{4}, u_{6}, u_{7}\right\},\left\{u_{5}\right\} \cup V^{\prime}\right\}$ is an upper domatic partition of $G$ with $V^{\prime}=V(G) \backslash\left\{u_{1}, u_{2}, \ldots, u_{7}\right\}$ proving that $D(G)=5$. 

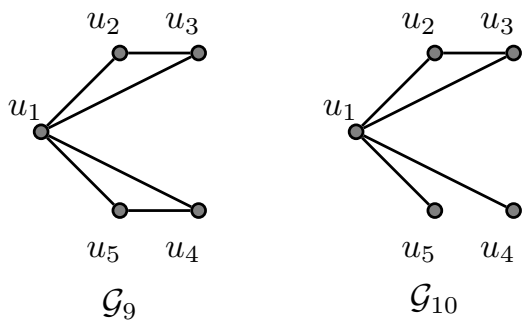

Figure 6: The graphs $\mathcal{G}_{9}$ and $\mathcal{G}_{10}$.

Case 6: $G\left[N\left[u_{1}\right]\right]=\mathcal{G}_{11}$, as shown in Figure 7.

Note that $\mathcal{G}_{11}$ is acyclic. If $G$ contains a $C_{3}$, then $G$ also contains one of the graphs $\mathcal{G}_{i}$, where $1 \leq i \leq 10$ as a subgraph. Hence it suffices to consider the case when $G$ is $C_{3}$-free. We now examine the adjacency relation between the vertices of $N^{1}\left(u_{1}\right)$ and $N^{2}\left(u_{1}\right)$.

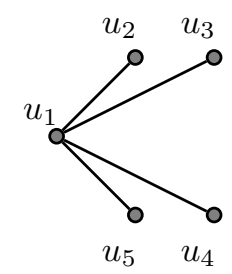

Figure 7: The graph $\mathcal{G}_{11}$.

Subcase 6.1: $\left|N^{1}\left(u_{6}\right) \cap N^{1}\left(u_{1}\right)\right|=4$, for some $u_{6} \in N^{2}\left(u_{1}\right)$.

In this case, if there is a vertex $u_{7} \in N^{2}\left(u_{1}\right)$, such that $u_{7}$ is adjacent to at least two vertices in $N^{1}\left(u_{1}\right)$, say $u_{2}$ and $u_{3}$, then $\left\{\left\{u_{1}\right\},\left\{u_{2}\right\},\left\{u_{3}, u_{6}\right\},\left\{u_{4}, u_{7}\right\},\left\{u_{5}\right\} \cup V^{\prime}\right\}$, where $V^{\prime}=V(G) \backslash\left\{u_{1}, u_{2}, \ldots, u_{7}\right\}$, is an upper domatic partition of $G$ proving that $D(G)=5$. If every vertex in $N^{2}\left(u_{1}\right)$ except $u_{6}$ is adjacent to exactly one vertex in $N^{1}\left(u_{1}\right)$, then the partition $\left\{\left\{u_{1}\right\},\left\{u_{2}\right\},\left\{u_{3}, u_{6}\right\},\left\{u_{4}, u_{7}, u_{8}\right\},\left\{u_{5}\right\} \cup V^{\prime}\right\}$, where $u_{7}, u_{8} \in N^{2}\left(u_{1}\right)$ are adjacent to $u_{2}$ and $u_{3}$ respectively and $V^{\prime}=V(G) \backslash\left\{u_{1}, u_{2}, \ldots, u_{8}\right\}$, is an upper domatic partition of $G$ proving that $D(G)=5$.

Subcase 6.2: $\left|N^{1}\left(u_{6}\right) \cap N^{1}\left(u_{1}\right)\right|=3$, for some $u_{6} \in N^{2}\left(u_{1}\right)$.

Let $u_{4} \in N^{1}\left(u_{1}\right)$ be the vertex not adjacent to $u_{6}$. Consider another vertex $u_{7} \in N^{2}\left(u_{1}\right)$ adjacent to the vertex $u_{2}$. Let $u_{8} \in N^{3}\left(u_{1}\right)$ be a vertex adjacent to $u_{7}$. Then, the partition $\pi=\left\{\left\{u_{1}\right\},\left\{u_{2}\right\},\left\{u_{3}, u_{7}\right\},\left\{u_{4}, u_{6}, u_{8}\right\},\left\{u_{5}\right\} \cup V^{\prime}\right\}$, where $V^{\prime}=V(G) \backslash\left\{u_{1}, u_{2}, \ldots, u_{8}\right\}$, is an upper domatic partition of $G$ proving that $D(G)=5$.

Subcase 6.3: $\left|N^{1}\left(u_{6}\right) \cap N^{1}\left(u_{1}\right)\right|=2$, for some $u_{6} \in N^{2}\left(u_{1}\right)$.

Let $u_{3}$ and $u_{5}$ be the vertices in $N^{1}\left(u_{1}\right)$ not adjacent to $u_{6}$ implying that $u_{2}, u_{4} \in N^{1}\left(u_{1}\right)$ are adjacent to $u_{6}$. If there exists a vertex $u_{7} \in N^{2}\left(u_{1}\right)$ that is adjacent to both $u_{2}$ and $u_{3}$, then the partition $\pi=\left\{\left\{u_{1}\right\},\left\{u_{2}\right\},\left\{u_{3}, u_{6}\right\},\left\{u_{4}, u_{7}\right\},\left\{u_{5}\right\} \cup V^{\prime}\right\}$, where $V^{\prime}=V(G) \backslash\left\{u_{1}, u_{2}, \ldots, u_{7}\right\}$, is an upper domatic partition of $G$ proving that $D(G)=5$. Else if $u_{7} \in N^{2}\left(u_{1}\right)$ is adjacent to $u_{2}$ but not $u_{3}$, then there exists $u_{8} \in N^{2}\left(u_{1}\right)$ such that $u_{3} u_{8} \in E(G)$, so that the partition $\left\{\left\{u_{1}\right\},\left\{u_{2}\right\},\left\{u_{3}, u_{6}\right\},\left\{u_{4}, u_{7}, u_{8}\right\},\left\{u_{5}\right\} \cup\right.$ $\left.V^{\prime}\right\}$, where $V^{\prime}=V(G) \backslash\left\{u_{1}, u_{2}, \ldots, u_{8}\right\}$, is an upper domatic partition of $G$ proving that $D(G)=5$. In case $u_{4}$ is adjacent to both $u_{6}$ and $u_{7}$, the vertex $u_{8}$ is chosen such that $u_{4} u_{8} \notin E(G)$.

Subcase 6.4: $\left|N^{1}(u) \cap N^{1}\left(u_{1}\right)\right|=1$, for each $u \in N^{2}\left(u_{1}\right)$.

Consider the vertices $u_{6}, u_{7} \in N^{1}\left(u_{2}\right), u_{8} \in N^{1}\left(u_{3}\right)$ and $u_{9} \in N^{1}\left(u_{6}\right)$ along with $N^{1}\left(u_{1}\right)=\left\{u_{2}, u_{3}, u_{4}, u_{5}\right\}$. Then, either the partition $\left\{\left\{u_{1}\right\},\left\{u_{2}\right\},\left\{u_{3}, u_{6}\right\},\left\{u_{4}, u_{7}, u_{8}\right\},\left\{u_{5}, u_{9}\right\} \cup V^{\prime}\right\}$ or the partition $\left\{\left\{u_{1}\right\},\left\{u_{2}\right\},\left\{u_{3}, u_{6}\right\},\left\{u_{4}, u_{7}, u_{8}, u_{9}\right\},\left\{u_{5}\right\} \cup V^{\prime}\right\}$, where $V^{\prime}=V(G) \backslash\left\{u_{1}, u_{2}, \ldots, u_{9}\right\}$, is an upper domatic partition of $G$ of cardinality five, the former being the case when $u_{6}$ is adjacent to $u_{8}$.

Thus, in all the six cases we have demonstrated the existence of an upper domatic partition of cardinality five of the graph $G$, proving that $D(G)=5$. This completes the proof.

Corollary 2.3. If $G$ is a 4-regular graph such that $G \neq G_{3,2}$, then $D(G)=5$.

Remark 2.1. There exist r-regular graphs of same order having different upper domatic numbers. 


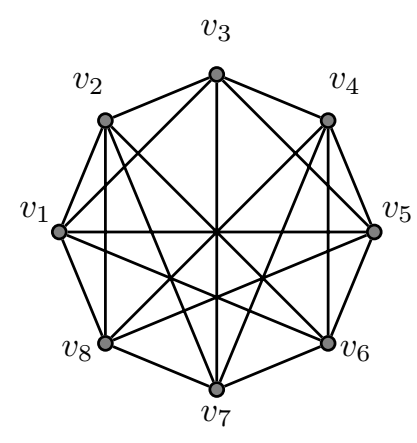

(a) $G_{1}$

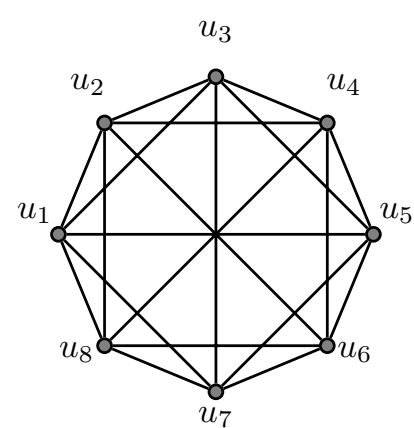

(b) $G_{2}$

Figure 8: Regular graphs of same order with different upper domatic numbers.

The partition $\pi_{1}=\left\{\left\{v_{5}, v_{6}, v_{7}\right\},\left\{v_{4}, v_{8}\right\},\left\{v_{1}\right\},\left\{v_{2}\right\},\left\{v_{3}\right\}\right\}$ is an upper domatic partition of the graph $G_{1}$. If $D\left(G_{1}\right)>5$, then a $D$-partition of $G_{1}$ contains at least four singleton sets, but it can be verified that $G_{1}$ contains no $K_{4}$. Therefore, $D\left(G_{1}\right)=5$. It can be easily seen that $D\left(G_{2}\right)=6$ with the $D$-partition $\pi_{2}=\left\{\left\{u_{2}, u_{6}\right\},\left\{u_{4}, u_{8}\right\},\left\{u_{1}\right\},\left\{u_{3}\right\},\left\{u_{5}\right\},\left\{u_{7}\right\}\right\}$.

Remark 2.2. $G_{3,2}$ is the graph with least order such that $D\left(G_{3,2}\right)<\delta\left(G_{3,2}\right)+1$, which leads to the following conjecture.

Conjecture. If a graph $G$ is $G_{3,2}$-free, then $D(G) \geq \delta(G)+1$.

Theorem 2.7. If $G$ is a 5-regular graph of girth at least five, then $D(G)=6$.

Proof. Let $G$ be a 5-regular graph of girth at least five. We prove this result by employing a colouring protocol where the set of colour classes form an upper domatic partition of the graph $G$. Choose an induced cycle $C_{k}$, where $k \geq 5$. Colour four consecutive vertices of the cycle with colours $3,1,2$ and 3 respectively and the remaining vertices of the cycle with colour 6. Further, assign colours 4, 5 and 6 to vertices lying outside $C_{k}$ and are adjacent to the vertices that have been coloured 1, 2 and 3 as shown in Figure 9.

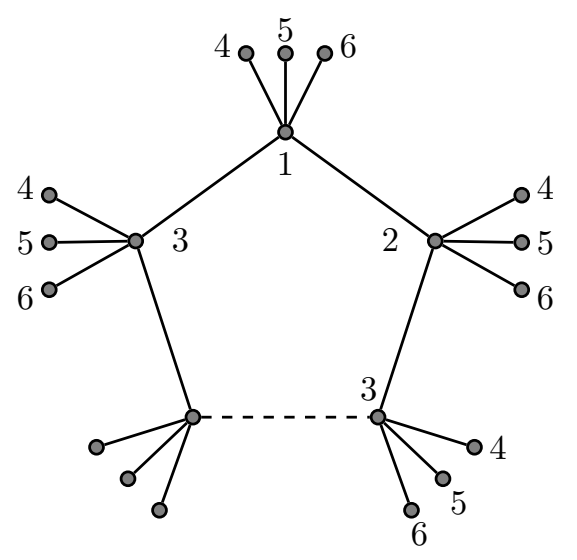

Figure 9: A subgraph of a 5-regular graph $G$ of girth at least 5.

Next colour the neighbours of vertices coloured 4 in such a way that at least two of these vertices are assigned colours 5 and 6. Note that in case there is adjacency among the vertices that have been already coloured, this condition automatically follows. Finally, colour all the remaining vertices with colour 6 . The resulting vertex partition of colour classes is an upper domatic partition of cardinality six, thus proving that $D(G)=6$.

Next, we discuss the upper domatic number of the complement $\overline{C_{n}}$ of a cycle $C_{n}$. Note that the complement of a cycle of order $n$ is an $(n-3)$-regular graph whose clique number is $\omega\left(\overline{C_{n}}\right)=\left\lfloor\frac{n}{2}\right\rfloor$.

Theorem 2.8. For the cycle $C_{n}, D\left(\overline{C_{n}}\right)=\left\lfloor\frac{3 n}{4}\right\rfloor$.

Proof. Consider the cyclic labelling of the vertices of $\overline{C_{n}}$ where $u_{i}$ is not adjacent to $u_{i-1(\bmod n)}$ and $u_{i+1(\bmod n)}$, see the labelling of $\overline{C_{8}}$ shown in Figure 10. Depending on the value of $n$, the vertex set is partitioned as follows.

Case 1: When $n(\bmod 4)=1$, partition the vertex set of $\overline{C_{n}}$ in the following manner, where $t=\left\lfloor\frac{n}{2}\right\rfloor$. 


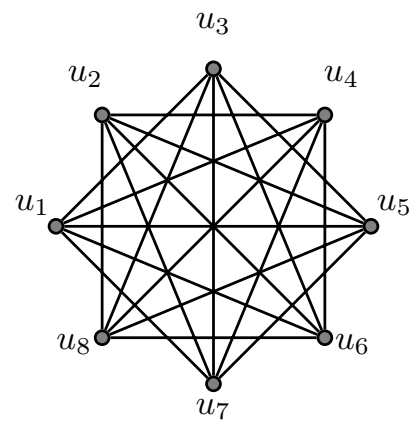

Figure 10: The graph $\overline{C_{8}}$.

$$
V_{i}= \begin{cases}\left\{u_{2 i-1}\right\}, & \text { if } 1 \leq i \leq t, \\ \left\{u_{2(i-t)}, u_{2(i-t)+t}\right\}, & \text { if } t+1 \leq i \leq\left\lfloor\frac{3 n}{4}\right\rfloor-1, \\ \left\{u_{t}, u_{n-1}, u_{n}\right\}, & \text { if } i=\left\lfloor\frac{3 n}{4}\right\rfloor .\end{cases}
$$

Case 2: When $n(\bmod 4)=2$, the following vertex partition is considered, where $t=\left\lfloor\frac{n}{2}\right\rfloor$.

$$
V_{i}= \begin{cases}\left\{u_{2 i-1}\right\}, & \text { if } 1 \leq i \leq t, \\ \left\{u_{2(i-t)}, u_{2(i-t)+t-1}\right\}, & \text { if } t+1 \leq i \leq\left\lfloor\frac{3 n}{4}\right\rfloor-1, \\ \left\{u_{t-1}, u_{n-2}, u_{n}\right\}, & \text { if } i=\left\lfloor\frac{3 n}{4}\right\rfloor .\end{cases}
$$

Case 3: When $n(\bmod 4)=3$, we consider the following partition where $t=\left\lfloor\frac{n}{2}\right\rfloor$.

$$
V_{i}= \begin{cases}\left\{u_{2 i-1}\right\}, & \text { if } 1 \leq i \leq t, \\ \left\{u_{2(i-t)}, u_{2(i-t)+t+1}\right\}, & \text { if } t+1 \leq i \leq\left\lfloor\frac{3 n}{4}\right\rfloor-1, \\ \left\{u_{t+1}, u_{n}\right\}, & \text { if } i=\left\lfloor\frac{3 n}{4}\right\rfloor .\end{cases}
$$

Case 4: When $n(\bmod 4)=0$, partition $V\left(\overline{C_{n}}\right)$ in the following manner, where $t=\left\lfloor\frac{n}{2}\right\rfloor$.

$$
V_{i}= \begin{cases}\left\{u_{2 i-1}\right\}, & \text { if } 1 \leq i \leq t, \\ \left\{u_{2(i-t)}, u_{2(i-t)+t}\right\}, & \text { if } t+1 \leq i \leq\left\lfloor\frac{3 n}{4}\right\rfloor .\end{cases}
$$

The partitions thus obtained in all these cases are upper domatic partitions. The sets $V_{1}, V_{2}, \ldots, V_{t}$ are singleton sets and contain the vertices $u_{1}, u_{3}, \ldots, u_{2 t-1}$, respectively, which together induce a clique of $\overline{C_{n}}$. In $\overline{C_{n}}$, any set $\left\{u_{i}, u_{j}\right\}$, where $j \notin\{(i-2) \bmod n,(i+2) \bmod n\}$ is a dominating set, hence the remaining sets in the partition are dominating sets. Since $\omega\left(\overline{C_{n}}\right)=t$, from Theorem 2.3 it follows that

$$
D\left(\overline{C_{n}}\right)=\left\lfloor\frac{3 n}{4}\right\rfloor
$$

\section{Acknowledgement}

We gratefully acknowledge the valuable suggestions of referees that helped us in improving this article.

\section{References}

[1] E. J. Cockayne, S. T. Hedetniemi, Towards a theory of domination in graphs, Networks 7 (1977) 247-261.

[2] F. Harary, Graph Theory, Addison-Wesley, Reading, 1969.

[3] T. W. Haynes, J. T. Hedetniemi, S. T. Hedetniemi, A. McRae, N. Phillips, The upper domatic number of a graph, AKCE Int. J. Graphs Comb. 17 (2020) 139-148.

[4] S. T. Hedetniemi, P. Slater, T. W. Haynes, Fundamentals of Domination in Graphs, CRC Press, Boca Raton, 1998.

[5] L. C. Samuel, M. Joseph, New results on upper domatic number of graphs, Commun. Comb. Optim. 5 (2020) 125-137.

[6] D. B. West, Introduction to Graph Theory, Prentice Hall, Upper Saddle River, 1996. 